\title{
The Electronic Kiosk \\ Accessing Newspapers with Electronic Media
}

\author{
Arnold Schneider, Cleto Pescia \\ Swiss Federation of the Blind and Visually Impaired
}

Contents

1. Abstract

2. Introduction

3. Information acquisition and preparation

4. Distribution

5 . The on-line reading program

6. Statistics - Graphs and Comments

7. Evolution of the project

8. Graphs

\section{Abstract}

This paper presents a project which has been initiated in Switzerland in 1992 to provide people who are blind or visually impaired with daily newspapers. At present newspapers in Italian and German language are available. Articles are sent to a host computer from where the readers can download the files they want to read. An especially developed reading program enables the reader to select the articles of his or her interest.

\section{Introduction}

As everywhere in the world blind people in Switzerland were thinking about a suitable way to access printed information sources with electronic media. The focus was put on daily papers, because this kind of information cannot be made available with tape recordings or in braille. We had knowledge of the project in Sweden [1], the research activity of TIDE [2], the project in Frankfurt Germany and of the project of the Italian Association of the Blind. They started in 1992 a project in which "La Stampa di Torino" was sent as ASCII file over a TV channel (Teletext or Videotext depending on local designations of the technique allowing the transfer of text information over a TV channel).

The blind people in the Italian speaking region of Switzerland had access to this innovative information source and after a short time they decided to start a local project. Using a TV channel is a complicated organizational problem and the transfer is very slow. So they decided to use a private host computer for data transfer from the editing office to the blind readers. On October 15, 1992 the "Edicola Elettronica" (the electronic kiosk) opened its electronic doors. All those blind people equipped with a PC and an appropriate output medium (speech synthesizer or braille display) could access two local newspapers every day. In addition they also could access "La Stampa 
di Torino" and 3 monthly magazines. Today about 30 persons are using regularly this service. For those who could not afford the cost for the required equipment, a special funding project has been started.

This beautiful and very valuable realization has motivated members of the Swiss Federation of the Blind in the German and French speaking areas of Switzerland to extend the electronic kiosk to their language and region. The break-through was reached end of 1993 when the "Neue Zürcher Zeitung" (NZZ), one of the most important papers published in German language in Switzerland accepted to make their issues available to blind readers in an appropriate form.

\section{Information acquisition and preparation}

The data files which contain the articles of an issue are collected by the editing office and sent via modem as one package to a host computer of EuroCom, a specialized company located in Lugano. The format of the data files usually varies from paper to paper, for the wordprocessing and document management systems used by the various publishers differ from one another. However, most papers use some kind of "mark-up language" to catalog the data for their internal needs, and therefore it is possible to easily isolate the relevant information about an article, like, for instance, the topic it belongs to. For each paper a special filter program is triggered as soon as the data files have been stored on the EuroCom host. This program processes the paper's data, filtering out the unnecessary information in a first step and then generating the electronic kiosk version of the paper. Each article is given a classification code, so the information can be grouped in 12 to 15 topics. This classification allows an easy selection of matter of interest in the reading program. Index files are generated in order to give fast access to a specific piece of information.

\section{Distribution}

The articles and index files are compressed before they are made available to the readers, in order to minimize transfer time and cost. Using a special communication program, the readers can dial in the system and download those parts of the paper they are interested in. The selection process happens off-line, before the actual connection takes place. This reduces the overhead of information to be exchanged during a connection, and helps in keeping the transmission costs low. The full transfer of an NZZ issue (about 180 to $250 \mathrm{~KB}$ ) takes 4 to 5 minutes at 9600 bps and costs less than 1 Swiss Frank per day.

\section{The on-line reading program}

This program has been especially developed in order to provide an easy and comfortable access to the text files transferred to the user over the telephone line. This program is menu driven and specifically designed to be used with speech synthesizers (simple presentation, full word menu items). At the top level about 12 topics are proposed to the user, e.g. home events, foreign events, culture, sports, tourism etc. Having selected one of these topics, the user obtains the list of articles pertaining to the chosen topic. The user can navigate with the cursor keys and select 
an article with the ENTER key. The article is then presented according to the selected reading mode. There is a "page by page mode" as in standard text editors, and there is a "continuous reading mode" especially designed for users of speech synthesizers. The whole article is sent as a continuous data flow to the output channel. The user can listen to the text output without the need of hitting a key after a fixed number of lines; but he may stop and restart reading at any time by hitting the space bar.

In addition the program offers a library function. All transferred issues can be stored and accessed later through a menu. Individual articles can be extracted from an issue and stored in a special archive directory.

\section{Statistics - Graphs and Comments}

The electronic kiosk has undergone beta testing in the period from April, 1 to June, 30,1994 . Only a few comments can be reasonably made based on the statistics gathered during these 13 testing weeks. The graphs are illustrated on last page.

Graphs No. 1 "No. of Calls in Each Test Day" shows an erratic behaviour of callers. As we will see in the following graph, this is due mainly to the fluctuating interest of readers in newspapers depending on the day of the week. Graph No. 2 "Daily Frequency of Calls" reveals a peak interest on Monday (over 120 calls), a still intense but somewhat lessened interest from Tuesday through Thursday (around 110 calls a day), and a definitely declining interest with the approach of the week end (from 95 to 70 , to 38 calls from Friday through Sunday).

A tendency which is independent on the special test conditions, is probably the "Hourly Frequency of Calls" during the day, as depicted in Graph No. 3. As it probably happens to sighted people, the preferred moment to buy the newspaper is in the early moment. In fact, Graph No. 3 shows the maximum peak of over 80 calls between seven and eight o'clock. We meet the second peek just before lunch (47 calls between 11 and 12 o'clock) and after lunch (57 calls between one and two PM). The third major peak is found in the evening, from eight to eleven o'clock, with 37 calls between eight and nine, 51 calls between nine and ten, and 42 calls between ten and eleven PM. But speaking in general, and except for the around-noon peaks, the peaks correspond basically with the low-tarif telephone time, which in Switzerland goes from 5 to $7 \mathrm{PM}$ and from $9 \mathrm{PM}$ to $8 \mathrm{AM}$. However, newspapers are downloaded during the whole day. Only from one o'clock in the night to five o'clock in the morning there has never been a hook up.

\section{Evolution of the project}

The next goal is to obtain a similar result in the French speaking area. Discussions are progressing and we hope to have access to a weekly magazine in a very short time. The main problem is in general at the operational level. Not all editing offices are preparing the articles in a way which allows relatively simple extraction of pure ASCII files. There is also the problem to define the people who are responsible for the data transfer to the kiosk host computer.

With a growing group of users of the electronic kiosk more requirements for the reading program will arise. The Swiss Federation of the Blind is prepared to sponsor 
further developments in this field. The leading staff is convinced that this project is a very significant contribution to the social and cultural integration of visually impaired people.

\section{Graphs}

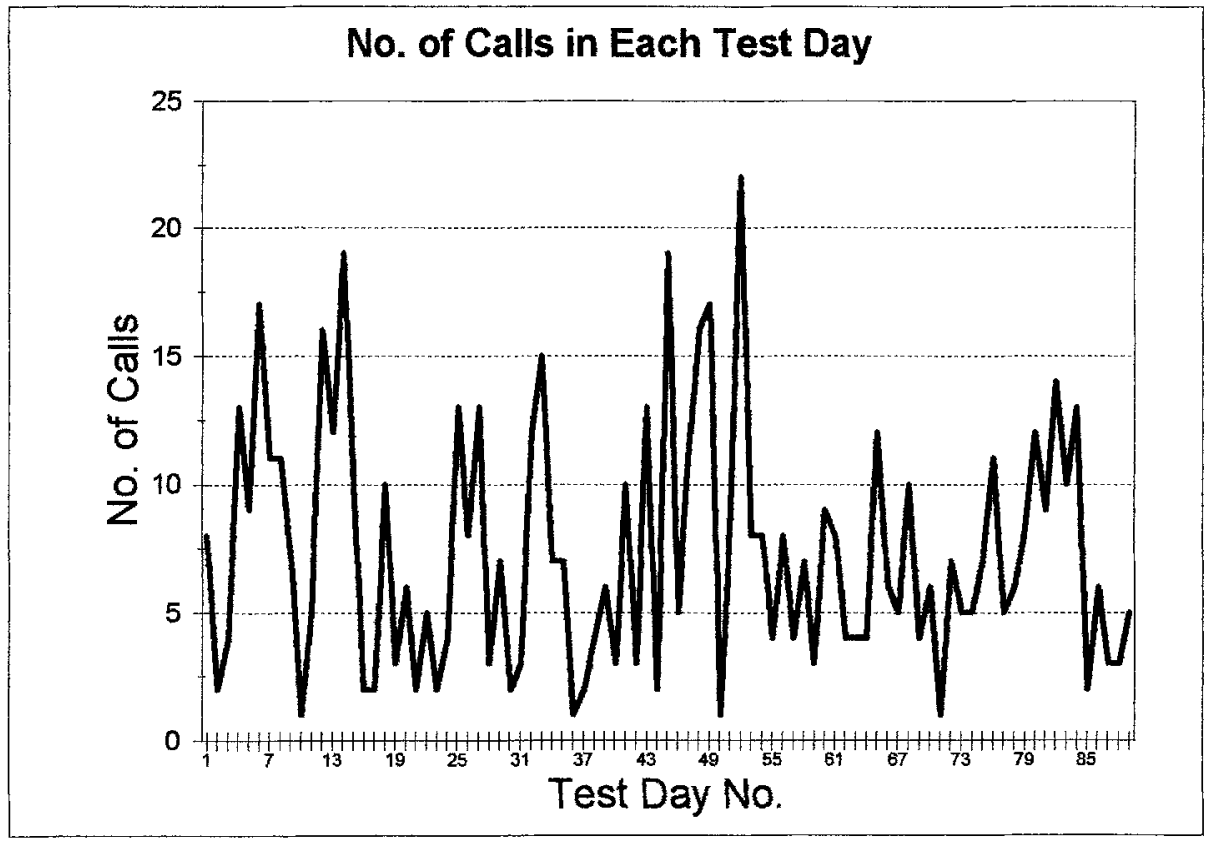

Graph 1. No. of Calls in Each Test Day

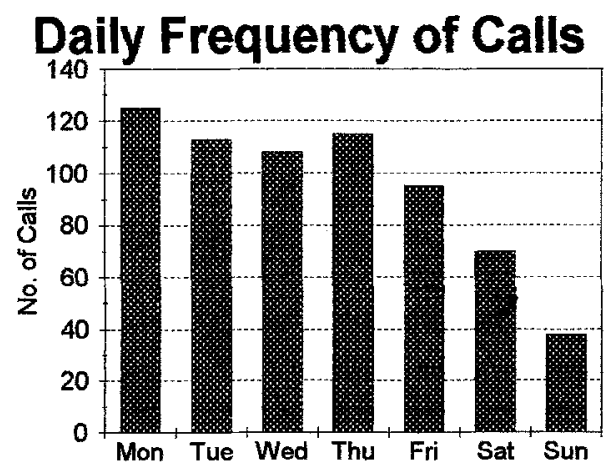

Graph 2. Daily Frequency of Calls 


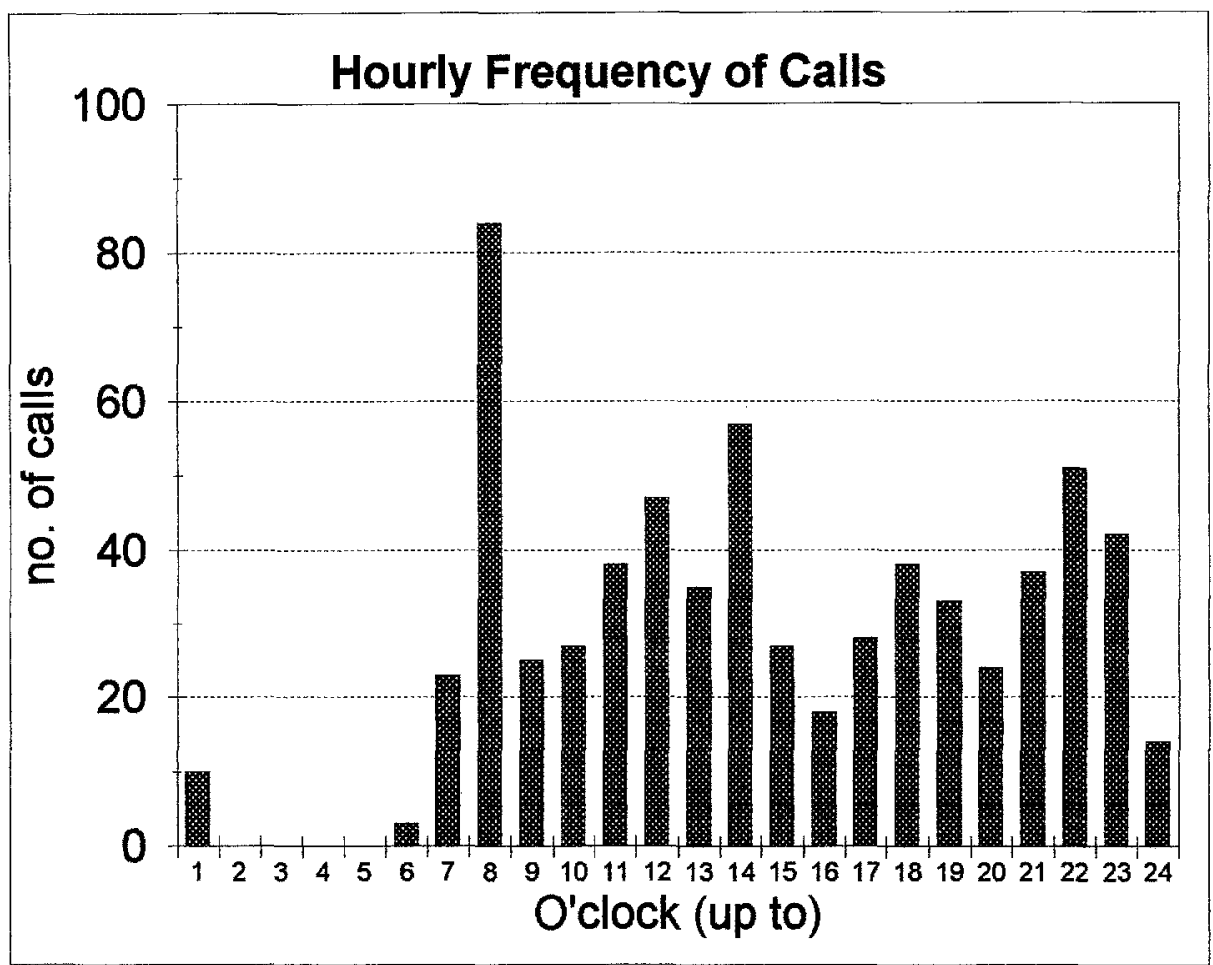

Graph 3. Hourly Frequency of Calls

\section{References}

[1] Henryk Rubinstein and Aleksander Ratz: Radio-Distributed Talking Electronic Newspaper for the Blind - an Everyday Service; procedings of the World Congress on Technology, December 1-5 1991, Washington D.C.

[2] TIDE: "Technology for the socio-economic Integration of the Disabled and Elderly People".: Project CAPS: Communication and Access to Information for Persons with Special Needs, final report December 1991 - March 1993. 